\title{
Experienced survey participants, biased responses? - The example of cognitive reflection
}

\section{Sven Grüner}

Institute of Agricultural and Nutritional Sciences

Martin Luther University Halle-Wittenberg

Halle (Saale), Germany

sven.gruener@landw.uni-halle.de

* corresponding author

\section{Norbert Hirschauer}

Institute of Agricultural and Nutritional Sciences

Martin Luther University Halle-Wittenberg

Halle (Saale), Germany

norbert.hirschauer@landw.uni-halle.de

\section{Acknowledgments}

(1) The overall project has been approved by the institutional review board of the Martin Luther University Halle-Wittenberg.

(2) The financial support of the Deutsche Forschungsgemeinschaft (DFG, German Research Foundation 388911356) is gratefully acknowledged. 


\title{
Experienced survey participants, biased responses? - The example of cognitive reflection
}

\begin{abstract}
The Cognitive Reflection Test (CRT) is used in behavioral studies to describe individuals as intuitive or reflective. In recent years, behavioral scientists have increasingly recruited participants on online platforms. Besides convenience, apt platform recruitment is believed to increase validity and facilitate more meaningful inferences towards the population of interest compared to studies based on students. But particularly with regard to CRT, there is an oftenunderestimated drawback: "experienced clickers" might systematically distort findings.
\end{abstract}

Keywords: cognitive reflection test; professional clickworkers; measurement error JEL: C91; D01

\section{Introduction}

Over the past decades, external validity has been a major concern of behavioral scientists, who commonly used university students in their studies. Despite the advantages of student subject pools (e.g. low opportunity costs, high availability), they are often systematically unrepresentative of the population of interest such as a country's residents. Age or working experience are obvious examples. Therefore, behavioral scientists are increasingly trying to access more informative subject pools through online platforms that build sampling frames by recruiting volunteers from the "general population" (Gosling and Mason 2015). Individual study participants are selected from those sampling frames and receive a small fee when they complete a survey. A validity problem could arise when a substantial number of professional clickworkers are recruited who frequently participate in studies (Hillygus et al. 2014). Their familiarity with standard tasks such as those in a Cognitive Reflection Test (CRT) might distort the findings.

The CRT, which has been introduced by Frederick (2005), is used to measure to what extent people can be categorized as being intuitive or reflective. Within a CRT, people are asked questions that invite intuitive answers that are wrong but also enable participants to identify correct answers after conscious reflection. The following question provides an illustration: "A bat and a ball cost $€ 1.10$ in total. The bat costs $€ 1.00$ more than the ball. How much does the ball cost?" (wrong answer $€ 0.10$, correct answer $€ 0.05$ ). The CRT is theoretically anchored in dual process theories, according to which the functioning of the human brain can virtually be distinguished into two systems: System 1 is fast and automatic, and System 2 is slow and deliberative (Stanovich and West 2000; Kahneman 2011).

A CRT might be fraught with measurement error that causes distortions when people are familiar with the task. While some participants might have seen the task before without having made a particular attempt to learn about its solution, others might have actively searched or read up on the correct answer. Besides the familiarity with concrete tasks, survey experience in general might influence the performance in a CRT (i.e., the number of correct answers). With this in mind, the paper investigates the following research questions:

(1) Do individuals who have seen the concrete CRT tasks before perform better in CRT? 
(2) Do individuals with generally more experience in surveys perform better in CRT?

To answer these questions, we carry out Frederick's (2005) CRT as part of a larger survey concerned with attitudes and characteristics of the general population of Germany. The online platform Respondi was used to implement the survey.

Our study is similar to Chandler et al. (2019) who also analyzed the impact of prior exposure to CRT on participants' performance in subsequent CRTs. Chandler et al. found that selfreported prior exposure to CRT is associated with higher performance. While they concerned themselves with the U.S. population and used Amazon Mechanical Turk (MTurk), we are interested in the German population and resort to Respondi. But we also use Frederick's CRT as a measurement device. Following Reed (2019), our study can thus be regarded as an "extension replication" that asks the question of whether the external validity of the Chandler et al. study extends to populations other than the one they investigated.

\section{Study design}

There are three variables of interest: On the one hand, there is (1) the number of correctly answered questions (“CRT-Score," ranging from 0 to 3) in Frederick's 2005 CRT (cf., Table 1) that is used to measure the extent of reflective thinking in individuals. On the other, there is (2) participants' prior exposure to CRT-specific tasks and (3) participants' prior experience with surveys. To measure exposure to CRT-specific tasks, participants were asked whether they had seen the questions in Table 1 or similar ones before (answering categories: no, unsure, yes). To elicit survey experience, we asked participants to indicate the number of prior participations (answer options: any number from 0 to 20 or more than 20). Before seeing the data, we decided to use the four response classes $0,1-10,11-20$, and $>20$.

Table 1. CRT-questions according to Frederick (2005)

\begin{tabular}{|l|c|}
\hline & $\begin{array}{c}\text { Correct } \\
\text { answer }\end{array}$ \\
\hline (1) A bat and a ball cost $€ 1.10$ in total. The bat costs $€ 1.00$ more than the ball. How much does the ball cost? _ $€$ & 0.05 \\
\hline $\begin{array}{l}\text { (2) If it takes } 5 \text { machines } 5 \text { minutes to make } 5 \text { widgets, how long would it take } 100 \text { machines to make } 100 \text { widgets? } \\
\text { minutes }\end{array}$ & 5 \\
\hline $\begin{array}{l}\text { (3) In a lake, there is a patch of lily pads. Every day, the patch doubles in size. If it takes } 48 \text { days for the patch to } \\
\text { cover the entire lake, how long would it take for the patch to cover half of the lake? __ days }\end{array}$ & 47 \\
\hline
\end{tabular}

(a) The correct answer was not shown to the participants.

\section{Empirical findings}

A total of 546 participants joined the survey from December 2021 to January 2022. Slightly less than $50 \%$ indicated being female. On average, subjects are 45 years old (for more details, see Appendix 1).

The mean CRT-Score was 0.98 ( $\mathrm{SD}=1.04)$ (see, Table 2). About two-fifths of the participants indicated not having seen questions similar to those in Table 1 before. A comparable share indicated having already seen such questions. Approximately one fifth was not sure. Only a small fraction of participants $(5.1 \%)$ had not had joined surveys in the last 5 years, but over two thirds $(67.8 \%)$ had participated more than 20 times. 
Table 2. Participants' CRT-Score and clicking experience ( $\mathrm{N}=546)$

\begin{tabular}{|l|l|l|}
\hline CRT-Score (number of correct answers; possible range from 0 to 3) & \multicolumn{1}{|c|}{0.98} \\
\hline \multirow{2}{*}{ Have you seen the quiz questions you've just answered in the same or similar form before? } & No & \multicolumn{1}{|c|}{$41.6 \%$} \\
\cline { 2 - 3 } & Unsure & $19.4 \%$ \\
\cline { 2 - 3 } & Yes & $39.0 \%$ \\
\hline \multirow{2}{*}{ How many times have you participated in surveys in the last 5 years (not counting this survey)? } & 0 & \multicolumn{1}{|c|}{$5.1 \%$} \\
\cline { 2 - 3 } & $1-10$ & $18.0 \%$ \\
\cline { 2 - 3 } & $11-20$ & $9.2 \%$ \\
\cline { 2 - 3 } & $>20$ & $67.8 \%$ \\
\hline
\end{tabular}

Regarding research question 1, a comparison of group means shows that the CRT-Score substantially increases with participants' familiarity with CRT (upper part of Figure 1): the mean score of 1.02 in the "unsure-familiarity group" is 0.34 higher than the mean of 0.68 in the "no-familiarity group" $(p=.0034) .{ }^{1}$ And with 1.28 , the mean in the "familiarity group" is 0.26 higher than the mean in "unsure-familiarity group" ( $p=.0526)$. In line with what Chandler et al. (2019) found for a sample of subjects from the U.S. population, higher familiarity with CRTspecific tasks was also consistently associated with higher CRT-Scores in our sample from the German population.

Regarding research question 2, the lower part of Figure 1 shows that more experience in surveys in general is also associated with higher CRT-Scores: the mean CRT-Score of 1.06 in the most experienced group ( $>20)$ was by 0.35 higher than the score of 0.71 in the group with zero prior survey experience $(p=.0463)$. With scores of 0.84 and 0.82 , the means of the two groups with prior survey experience from 1-10 and from 11-20 were almost equal and they were located between those of the zero-experience and the most experienced group.

We may summarize that both prior exposure to CRT-specific tasks and experience with surveys in general are consistently associated with higher performance. But, as might have been expected, it seems that prior exposure to CRT is more relevant than having survey experience in general. ${ }^{2}$

\footnotetext{
${ }^{1}$ It must be observed that statistical inferential procedures are based on probability theory and presuppose a random process of data generation (e.g. random sampling). When there is no probabilistic data generation process, a meaningful inferential interpretation of sample statistics, such as $p$-values, as measures of sample effect sizes relative to sampling variation requires assuming that the sample under study is approximately a random sample from the population of interest (Hirschauer et al. 2021). Since this seems quite a heroic assumption for our sample, we do not focus on inferential statistics based on the standard error. Nonetheless, we display $p$-values (Wilcoxonrank sum) for those who find them generally informative as summary statistics of a dataset without explicitly questioning whether there is a chance model linking that dataset to the target population.

${ }^{2}$ In regression analysis, which was used as robustness check and which related prior exposure to CRT and survey experience in general with CRT-Scores, similar results were obtained. Regression results both without and with control variables can be found in Appendix 2.
} 
Figure 1. Relating experience with CRTs and surveys in general with $C R T$-scores $(\mathrm{N}=546)$

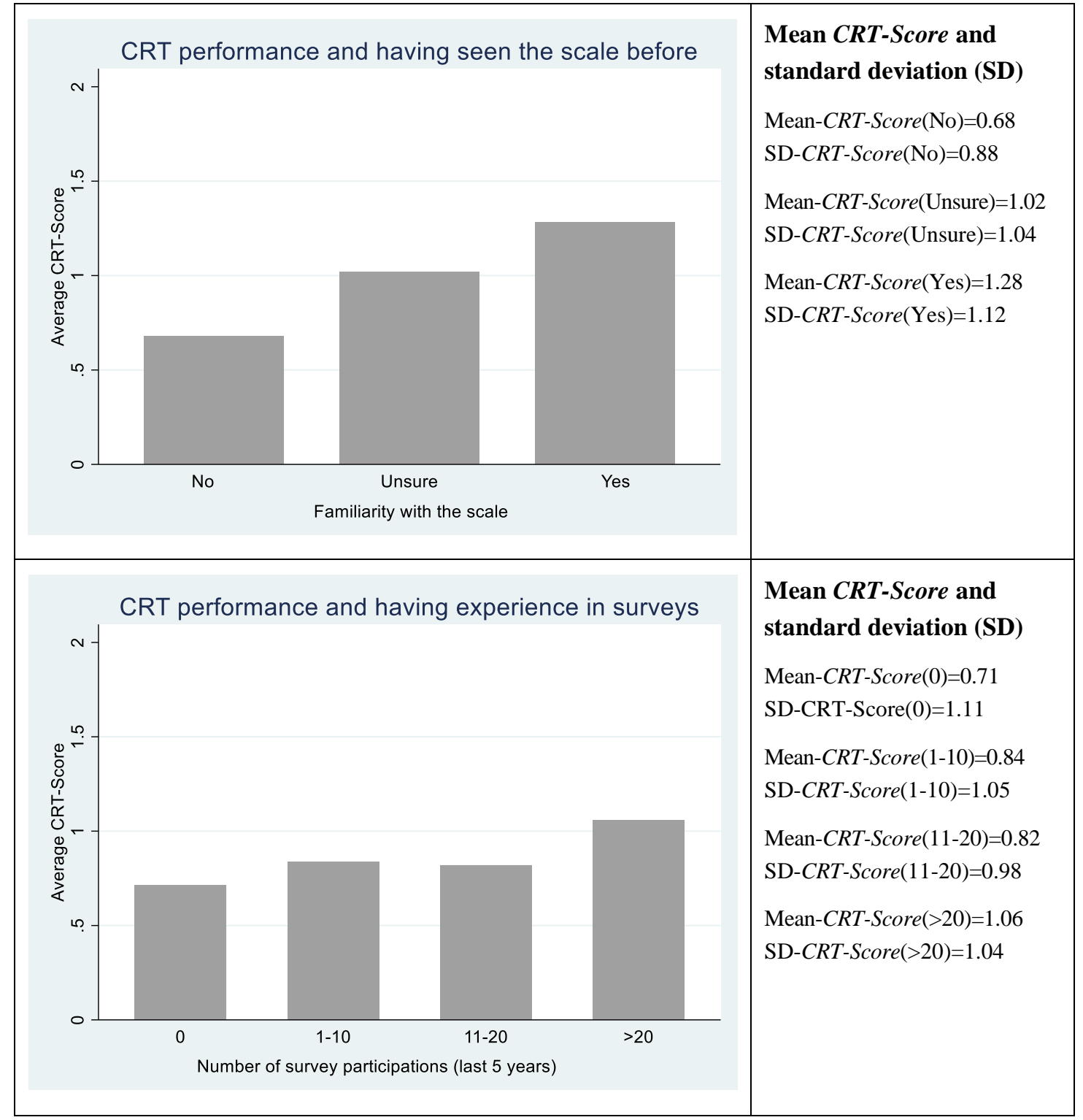

\section{Conclusions}

It is generally believed that it is preferable or even necessary for replication or longitudinal studies to use identical measurement devices (e.g. identical survey item wordings or identical experimental tasks) for identical theoretical constructs. In some research settings, this may be associated with drawbacks, however. For example, in recent years, researchers have increasingly recruited participants for follow-up studies from sampling frames such as those provided by online platforms (e.g. MTurk or Respondi). As a consequence, and contrary to the original intention, measurement problems may arise precisely because the same measurement device is used. This is the case when participants' answering behavior is sensitive to prior experiences. Using cognitive reflection as an example, we have shown that participants' prior exposure to CRT-specific tasks and survey experience in general systematically distort findings toward higher CRT-Scores. Sensitivity is also likely to be a relevant problem in other fields such as the elicitation of people's risk attitudes through multiple price lists or intelligence testing. Therefore, further research should generally investigate approaches that are able to 
mitigate measurement problems resulting from participants' sensitivity to prior experiences. Using alternative measurement devices that are unknown to participants seems to be one promising approach. This presupposes, however, that they "measure the same thing" and come to a similar assessment of the theoretical construct. Excluding participants with prior experience from samples seems to be another. This might even partly rehabilitate the use of student subject pools and, in particular, the use of undergraduate students without much prior survey experience. Of course, all these approaches have their own drawbacks and tradeoffs that must be considered. For example, while distortions by prior experience might be avoided by resorting to undergraduate students, their above-mentioned lacking representativeness of the general population might be too serious a tradeoff. It all depends on the specific research context and questions.

\section{References}

Chandler, J., Rosenzweig, C., Moss, A.J., Robinson, J., Litman, L. (2019): Online panels in social science research: Expanding sampling methods beyond Mechanical Turk. Behavior Research Methods 51: 2022-2038.

Frederick, S. (2005): Cognitive reflection and decision making. Journal of Economic Perspectives 19: 25-42.

Gosling, S. D., Mason, W. (2015): Internet research in psychology. Annual Review of Psychology 66: 877-902.

Hillygus, D.S., Jackson, N., Young, M. (2014): Professional Respondents in Non-Probability Online Panels. In: Online Panel Research: A Data Quality Perspective. M. Callegaro, R. Baker, J. Bethlehem, A.S. Göritz, J. A. Krosnick, P.J. Lavrakas (eds.), NJ: John Wiley \& Sons, Ltd., 219-337.

Hirschauer, N., Grüner, S., Mußhoff, O., Becker, C., Jantsch, A. (2021): Inference using nonrandom samples? Stop right there! Significance 18: 20-24.

Kahneman, D. (2011): Thinking Fast and Slow. New York: Farrar, Strauss, Giroux.

Reed, W.R. (2019): Takeaways from the Special Issue on The Practice of Replication. Economics 13(1): 20190013.

Stanovich, K.E., West, R.F. (2000): Individual differences in reasoning: implications for the rationality debate. Behavioral and Brain Sciences 23: 645-726. 


\section{Appendix}

\section{Appendix 1: Details on the participants}

\section{Description of participants $(\mathrm{N}=546)^{(a)}$}

\begin{tabular}{|l|l|c|c|}
\hline \multicolumn{2}{|l|}{} & Mean/Fraction & Std. Dev. \\
\hline & Female & $46.9 \%$ & - \\
\hline Gender(a) & Male & $52.8 \%$ & - \\
\hline Age & Other & $0.4 \%$ & 14.9 \\
\hline $\begin{array}{l}\text { Higher education (“Abitur” - general university } \\
\text { entrance qualification - or higher) }\end{array}$ & 45.2 & \\
\hline Happiness & (b) & $27.8 \%$ & 2.2 \\
\hline Risk attitude & 6.6 & 2.6 \\
\hline
\end{tabular}

(a) Quotas for gender and education were chosen to mimic the general population of Germany.

(b) Participants were asked to indicate their highest degree on a 7-point scale, ranging from 1 (no degree) to Ph.D. This scale was also used in the regression analysis. For simplification, we only display the share of participant with higher education.\#\#

(c) All things considered, how satisfied are you with your life? ( $0=$ not satisfied at all, $\ldots, 10=$ completely satisfied)

(d) How would you see yourself: Are you, in general, prepared to take risks, or do you try to avoid risks? $(0=$ not at all willing to take risks, $\ldots, 10=$ very willing to take risks)

\section{Appendix 2: Robustness check}

The following table depicts the marginal effects obtained in ordered logit regressions explaining the CRT-Score. Two specifications are provided: specification I addresses only participants' prior exposure to CRT-specific tasks and their experience with surveys in general. Specification II adds some controls. The results are in line with the findings described above. For example, an increase in the prior exposure to CRT reduces the probability of being wrong in all three questions (-0.117). And prior exposure to CRT is more relevant for explaining CRT-Scores than experience with surveys in general.

\section{Marginal effects obtained in OLogit regressions ${ }^{(a)}$}

\begin{tabular}{|l|c|c|c|c|}
\hline & \multicolumn{3}{|c|}{ Y=CRT-Score (CRT-questions answered correctly) } \\
\hline & 0 & 1 & 2 & 3 \\
\hline Specification I & & & & 0.051 \\
\hline Exposure to CRT-specific tasks & -0.117 & 0.015 & 0.051 & 0.021 \\
\hline Experience with surveys in general & -0.048 & 0.006 & 0.021 & \\
\hline Specification II & & & & 0.052 \\
\hline Exposure to CRT-specific tasks & -0.117 & 0.015 & 0.049 & 0.021 \\
\hline Experience with surveys in general & -0.046 & 0.006 & 0.019 & 0.013 \\
\hline Gender (female vs. male) & -0.029 & 0.004 & 0.012 & -0.000 \\
\hline Age & 0.000 & -0.000 & -0.000 & 0.046 \\
\hline Education & -0.103 & 0.013 & 0.043 & 0.005 \\
\hline Happiness & -0.012 & 0.002 & 0.005 & -0.001 \\
\hline Risk & 0.003 & -0.000 & -0.001 & \\
\hline
\end{tabular}

(a) Specification I: Prob $>$ chi2<0.0001, Pseudo R2=0.0291; specification II: Prob $>$ chi2 $<0.0001$, Pseudo R2=0.0708. For general limitations regarding distributional assumptions employed in statistical analysis that arise from violations of random data generation, see footnote 1 (Section 3). 